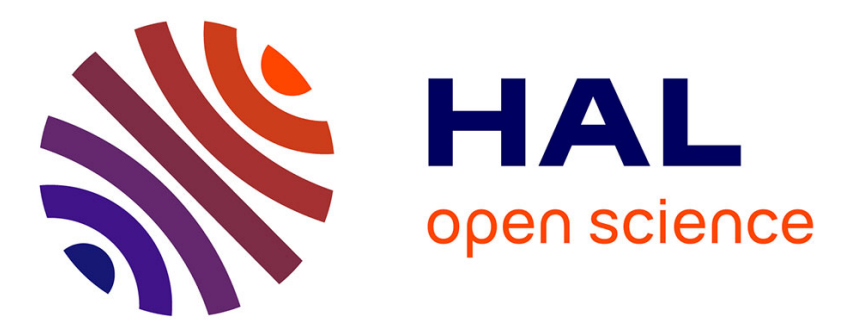

\title{
Les miroirs bicylindres et leur utilisation dans les spectromètres de masse magnétique à multiples passages \\ C. Berger, M. Baril
}

\section{To cite this version:}

C. Berger, M. Baril. Les miroirs bicylindres et leur utilisation dans les spectromètres de masse magnétique à multiples passages. Revue de Physique Appliquée, 1978, 13 (5), pp.271-281. 10.1051/rphysap:01978001305027100 . jpa-00244450

\section{HAL Id: jpa-00244450 https://hal.science/jpa-00244450}

Submitted on 1 Jan 1978

HAL is a multi-disciplinary open access archive for the deposit and dissemination of scientific research documents, whether they are published or not. The documents may come from teaching and research institutions in France or abroad, or from public or private research centers.
L'archive ouverte pluridisciplinaire HAL, est destinée au dépôt et à la diffusion de documents scientifiques de niveau recherche, publiés ou non, émanant des établissements d'enseignement et de recherche français ou étrangers, des laboratoires publics ou privés. 


\title{
LES MIROIRS BICYLINDRES ET LEUR UTILISATION DANS LES SPECTROMẼTRES DE MASSE MAGNÉTIQUE A MULTIPLES PASSAGES
}

\author{
C. BERGER
}

Laboratoire de Physique Industrielle, I. N. S. A. de Lyon, 69621 Villeurbanne Cedex, France

et

M. BARIL

Département de Physique et C. R. A. M., Université Laval, Québec, P. Qué., Canada

(Reçu le 30 décembre 1977, accepté le 30 janvier 1978)

\begin{abstract}
Résumé. - Les propriétés optiques des miroirs électrostatiques bicylindres ont été calculées grâce à la méthode mise au point par Recknagel il y a plus de quarante ans. Contrairement aux prétentions de l'auteur de la méthode, le nombre d'intervalles dans les trajectoires doit être assez grand si on veut atteindre une certaine précision. Les calculs montrent que le miroir est successivement divergent, convergent, divergent lorsque l'on augmente à partir de zéro le rapport du potentiel de répulsion à celui de la tension d'accélération des particules chargées incidentes. Le changement se produit pour les valeurs du rapport $V_{\text {rep. }} / V_{\text {acc., }}$ noté $V_{2} / V_{1}$ dans le texte, égales respectivement à $-0,0355$ et $-0,410$. Dans la deuxième partie, après un rappel du principe de fonctionnement du spectromètre de masse magnétique à multiples passages, on étudie l'imagerie dans le plan radial et l'acceptance dans le plan transversal. La troisième partie est consacrée à l'optimisation de la transmission qui ne dépend que du rapport $V_{2} / V_{1}$ et de la position du centre physique du miroir si les valeurs du rayon du prisme magnétique et de celui des cylindres du miroir ont été fixées à l'avance. Les résultats expérimentaux confirment l'analyse théorique et sont présentés dans la quatrième partie.
\end{abstract}

Abstract. - The optical properties of the two-cylinder mirror have been calculated by a method designed by Recknagel more than forty years ago. Precision is obtained only if the trajectories are divided in numerous intervals. Calculations have shown that the mirror is successively divergent, convergent and divergent when the ratio of the repulsion potential over the acceleration potential of the charged particles is increased from zero. The values of the ratio $V_{2} / V_{1}$ at which the change occurs are -0.0355 and -0.410 . In a second part, the principle on which the multipassage magnetic mass spectrometer rests is reviewed; also imaging in the radial plane and acceptance in the transversal plane are studied. The third part deals with the optimization of transmission through the mass spectrometer. The transmission depends only on two variables : the ratio $V_{2} / V_{1}$ and the position of the middle of the cylinders if the two other variables, the radius of curvature of the trajectories in the magnet and the radius of the cylinders, are fixed. The experimental results confirm the theoretical analysis and are presented in the fourth part.

Introduction. - Les miroirs électrostatiques ont été jusqu'à présent fort peu étudiés. Ceci est dû en partie à leur faible utilisation dans les appareils d'optique électronique, de même qu'aux difficultés de calcul que représente le rebroussement des trajectoires. Au contraire, le fonctionnement du spectromètre de masse magnétique à multiples passages $\left(\mathrm{SM}^{3} \mathrm{P}\right)$ repose sur les propriétés de réflexion de cet élément d'optique électronique pour multiplier à volonté le nombre de passages dans le prisme magnétique. Un choix judicieux des paramètres optiques du système permet d'accroître le pouvoir de résolution proportionnellement au nombre de passages. Dans les travaux antérieurs [1] sur les $\mathrm{SM}^{3} \mathrm{P}$ à éléments discontinus, seuls des miroirs ayant des propriétés focalisantes uniquement dans le plan radial ont été étudiés. L'utilisation de miroir bicylindre possédant une symétrie de révolution autour de l'axe optique, permet d'obtenir un système où la focalisation existe aussi dans le plan transversal. Comme on le montrera plus loin, on applique les conditions d'imagerie dans le plan radial, alors que dans le plan transversal on doit réaliser l'optimisation de la transmission, ce qui implique des perspectives et des calculs fort différents.

Nous étudierons dans une première partie les propriétés optiques du miroir bicylindre. Dans une seconde 
partie nous décrirons les caractéristiques, propriétés et exigences des $\mathrm{SM}^{3} \mathrm{P}$. La troisième partie sera consacrée à montrer comment les miroirs bicylindres peuvent être utilisés comme éléments réflecteurs dans les $\mathrm{SM}^{3} \mathrm{P}$. Les résultats expérimentaux seront analysés dans la quatrième partie. Puis nous tirerons les conclusions appropriées.

1. Le miroir bicylindre et ses propriétés optiques. 1.1 CARACTÉRISTIQUES GÉOMÉTRIQUES. - Le miroir bicylindrique est formé comme son nom l'indique en partie de deux cylindres de même axe, de même diamètre, séparés par un petit intervalle comme le montre la figure 1 . Les longueurs $\lambda_{1}$ et $\lambda_{2}$ des cylindres doivent

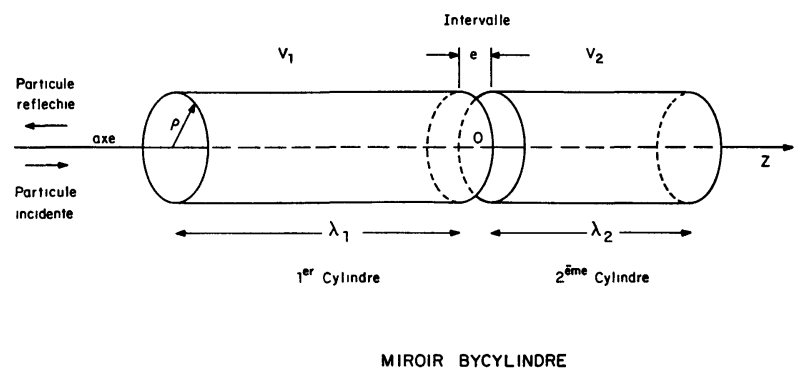

FIg. 1. - Le miroir bicylindre est constitué de deux cylindres de même axe $z$ de longueur respective $\lambda_{1}$ et $\lambda_{2}$ séparés par un intervalle de longueur $e$.

[The bicylinder mirror is made of two cylinders having the same axis. Their length are $\lambda_{1}$ and $\lambda_{2}$ and they are separated by a space of dimension $e$.]

être supérieures à trois fois le rayon $\rho$, si l'on veut pouvoir les considérer comme infiniment longs dans le calcul de la distribution de potentiel le long de l'axe des cylindres.

1.2 Principe de CAlCul Des trajeCtoires. - Le calcul des trajectoires repose habituellement sur l'un des deux principes suivants.

Le premier correspond à un découpage en pas de longueur constante suivant un axe et à une approche de la courbe réelle par itération à partir d'un arc de parabole.

Le deuxième principe utilise un découpage en intervalles de temps constants [2]; la courbe réelle est obtenue paramétriquement par itération à partir de développements limités des coordonnées en fonction du temps.

Cette seconde méthode présente l'avantage de pouvoir être utilisée sans aucune modification quand la trajectoire présente un point de rebroussement. Malheureusement le nombre de pas de calcul devient alors très grand.

Un travail de Recknagel [3] déjà ancien conduit à une autre possibilité de calcul des trajectoires paraxiales dans le cas de systèmes à symétrie de révolution cylindrique.
L'auteur a en effet montré que pour un potentiel sur l'axe de forme quadratique

$$
\varphi(0, z)=A+B\left(z-z_{\mathrm{m}}\right)^{2}
$$

l'équation de la trajectoire paraxiale est connue analytiquement en fonction des conditions initiales et des valeurs des constantes $A, B$ et $z_{\mathrm{m}}$.

Pour le potentiel particulier étudié

$$
\varphi(r, z)=A+B\left(z-z_{\mathrm{m}}\right)^{2}-\frac{B}{2} r^{2}
$$

où $r$ est la distance du point considéré à l'axe, l'expression

$$
k=w^{2}+\frac{B^{2}}{2} r^{2}
$$

est une constante.

Dans l'expression précédente $w$ représente la vitesse radiale $(\mathrm{d} r / \mathrm{d} t)$.

Lorsque les trajectoires sont situées dans le plan de symétrie l'intégration conduit à deux types de solutions selon le signe du coefficient $B$.

Si $B>0$.

$$
\begin{aligned}
& r=r_{0} \cos \alpha+\frac{w_{0}}{\sqrt{\frac{B}{2}}} \sin \alpha \\
& w=w_{0} \cos \alpha-\sqrt{\frac{B}{2}} r_{0} \sin \alpha
\end{aligned}
$$

avec

$$
\alpha=\frac{\sqrt{2}}{2} \ln \frac{z-z_{\mathrm{m}}+\sqrt{\left(z-z_{\mathrm{m}}\right)^{2}+\frac{A-k}{B}}}{z_{0}-z_{\mathrm{m}}-\sqrt{\left(z_{0}-z_{\mathrm{m}}\right)^{2}+\frac{A-k}{B}}}
$$

$r_{0}, w_{0}, z_{0}$ étant les conditions initiales.

Si $B<0$.

$$
\begin{aligned}
& r=r_{0} \cosh \beta+\frac{w_{0}}{\sqrt{-\frac{B}{2}}} \sinh \beta \\
& w=w_{0} \cosh \beta+\sqrt{-\frac{B}{2}} \sinh \beta
\end{aligned}
$$

$$
\begin{aligned}
\beta=\frac{\sqrt{2}}{2}\{\arcsin & {\left[\sqrt{\frac{-B}{A-k}}\left(z-z_{\mathrm{m}}\right)\right]-} \\
& \left.-\arcsin \left[\sqrt{\frac{-B}{A-k}}\left(z_{0}-z_{\mathrm{m}}\right)\right]\right\} .
\end{aligned}
$$

Un autre point intéressant du travail de Recknagel est d'avoir démontré que les équations données ci-dessus sont une approximation convenable au voisinage du point de rebroussement de trajectoires électroniques, bien que les conditions de Gauss ne soient plus respectées.

Le calcul des trajectoires a donc été programmé selon ce principe qui évite les itérations. Le choix du pas 
de progression $s$ est alors posé. Recknagel mentionnait à ce sujet que le partage de l'axe du système étudié en quelques intervalles seulement ( 3 ou 5) et le remplacement dans chaque intervalle du potentiel réel par une expression approchée de la forme :

$$
\varphi(z, 0)=A+B\left(z-z_{\mathrm{m}}\right)^{2}
$$

suffisaient à l'obtention de résultats corrects.

Nous sommes en désaccord avec l'auteur sur l'emploi d'un nombre restreint d'intervalles qui conduisent au moins pour deux types de systèmes, à des résultats complètement erronés malgré tout le soin apporté à la formulation approchée du potentiel et au respect des conditions aux limites à chaque changement d'intervalles. Les trajectoires à point de rebroussement sont encore plus sensibles au choix du pas. Et dans le cas plus particulièrement étudié ici, la nullité de la dérivée seconde du potentiel au point milieu $\mathrm{O}$ du système peut créer pour ce choix un impératif supplémentaire.

Le contrôle de la validité des résultats obtenus est basé d'abord sur une comparaison avec ceux résultant d'autres méthodes de calcul, puis sur les valeurs de la matrice du miroir équivalent ce dont on parlera ultérieurement.

La valeur adoptée pour le pas est 0,01 (l'unité étant le rayon des cylindres) lorsque le caractère convergent ou divergent est très affirmé. Dans le cas, où ce caractère est peu affirmé et où les résultats évoluent au moindre changement de conditions, il est préférable d'employer un pas plus faible. Enfin, lorsque le point de rebroussement se situe au voisinage du point $O$, il est nécessaire de choisir un pas dont la valeur varie selon la région pour des questions de précision dans les calculs.

1.3 CARACTÉRISTIQUeS DU MIROIR BICYLINDRE EN FONCTION DU RAPPORT DES TENSIONS APPLIQUÉES. Précisons tout d'abord quelques notations et conventions utilisées dans la suite du texte.

L'unité quand elle n'est pas précisée est égale à la valeur du rayon des cylindres formant le miroir.

L'origine $O$ sur l'axe est située dans le plan de séparation des deux cylindres. Le sens positif adopté est toujours celui de la propagation des particules incidentes. Les angles comptés à partir de la direction positive de l'axe sont évalués positivement dans le sens trigonométrique.

Avec ces conventions, le potentiel $\Phi$ en un point de l'axe de côté $z$ est alors donné par la formule approximative, largement utilisée [4],

$$
\Phi(0, z)=\frac{V_{1}+V_{2}}{2}+\left(V_{2}-V_{1}\right) \tanh \omega z
$$

dans laquelle $V_{1}$ est le potentiel du premier cylindre traversé par les particules, $V_{2}$ le potentiel du deuxième cylindre (cf. Fig. 1) et $\omega$ une constante de valeur 1,318.

1.4 DÉTERMINATION DES PROPRIÉTÉS OPTIQUES DU MIROIR A PARTIR DES TRAJECTOIRES. - Considérons un miroir (M) quelconque de sommet $\mathrm{S}$ et de centre $\mathrm{C}$. Soient $\left(\mathbf{P}_{0}\right)$ et $\left(\mathbf{P}_{\mathbf{i}}\right)$ les deux plans de référence respectivement des espaces objet et image (Fig. 2). Si un rayon

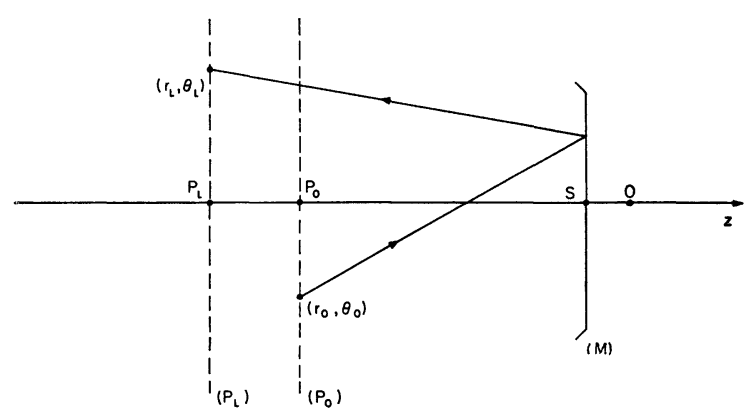

FIG. 2. - Représentation schématique du miroir. $P_{0}$ et $P_{i}$ sont respectivement le plan objet et le plan image, $\mathrm{S}$ le sommet du miroir et $\mathrm{O}$ le milieu de l'intervalle $e$ entre les cylindres.

[Schematic of the mirror showing object plane $P_{0}$, image plane $P_{i}$, summit $S$, and $O$ the middle of the spacing between the two cylinders.]

est défini par son ordonnée $r_{0}$ et sa pente $\theta_{0}$ au niveau de $\left(\mathrm{P}_{0}\right)$, les caractéristiques $\theta_{\mathrm{i}}$ et $\boldsymbol{r}_{\mathrm{i}}$ du rayon réfléchi au niveau de $\left(\mathrm{P}_{\mathrm{i}}\right)$ sont exprimées par :

$$
\begin{aligned}
\left(\begin{array}{l}
r_{\mathrm{i}} \\
\theta_{\mathrm{i}}
\end{array}\right)=\left(\begin{array}{ll}
1 & d_{\mathrm{i}} \\
0 & 1
\end{array}\right)\left(\begin{array}{cc}
1 & 0 \\
\frac{2}{\mathrm{CS}} & -1
\end{array}\right)\left(\begin{array}{cc}
1 & d_{0} \\
0 & 1
\end{array}\right) & \times \\
& \times\left(\begin{array}{l}
r_{0} \\
\theta_{0}
\end{array}\right)=M\left(\begin{array}{l}
r_{0} \\
\theta_{0}
\end{array}\right)
\end{aligned}
$$

avec

$$
d_{\mathrm{i}}=\overline{\mathrm{SP}_{\mathrm{i}}} \quad \text { et } \quad d_{0}=\overline{\mathrm{P}_{0} \mathrm{~S}} \text {. }
$$

Dans le cas particulier où les plans de référence sont confondus $\left(d_{0}=-d_{\mathbf{i}}=d\right)$, les coefficients de la matrice sont les suivants :

$$
M=\left(\begin{array}{ll}
A_{\mathrm{m}} & B_{\mathrm{m}} \\
C_{\mathrm{m}} & D_{\mathrm{m}}
\end{array}\right)=\left(\begin{array}{cc}
1-\frac{2 d}{\mathrm{CS}} & 2 d\left(1-\frac{d}{\mathrm{CS}}\right) \\
\frac{2}{\mathrm{CS}} & -\left(1-\frac{2 d}{\mathrm{CS}}\right)
\end{array}\right) .
$$

Pour l'étude effectuée ici, le plan de référence a été pris dans la région de champ nul à la cote $z_{\mathrm{d}}=-5$. Les coefficients de la matrice se calculent très simplement à partir des caractéristiques d'entrée et de sortie de deux trajectoires particulières (l'une correspondant à $r_{0}=0$, l'autre à $\theta_{0}=0$ ). Les caractéristiques du miroir se déduisent ensuite de $A_{\mathrm{m}}$ et $C_{\mathrm{m}}$.

Les résultats de nos calculs sont présentés au tableau I et à la figure 3. La gamme de rapport $V_{2} / V_{1}$ varie entre 0 et -1 . Nous avons agrandi la zone comprise entre $-0,3$ et $-0,38$ car elle correspond à la zone d'utilisation pour le type d'application à réaliser. Pour le calcul de la transmission il est utile de connaître les coefficients $A_{0}, B_{0}, C_{0}, D_{0}$. Il nous est apparu raisonnable de calculer leur valeur pour 


\section{TABLEAU I}

Caractéristiques du miroir bicylindre en fonction du rapport $V_{2} / V_{1}$ des potentiels appliqués aux cylindres.

L'unité de longueur est le rayon des cylindres

\begin{tabular}{|c|c|c|c|c|}
\hline$V_{2} / V_{1}$ & $\begin{array}{c}\text { Abscisse } \\
\text { du sommet }\end{array}$ & $\begin{array}{l}\text { Abscisse } \\
\text { du centre }\end{array}$ & Rayon & Natu \\
\hline$-0,01$ & $-1,588$ & 0,798 & 2,387 & $D$ \\
\hline$-0,05$ & 7,156 & $-\quad 0,106$ & 7,262 & \\
\hline$-0,1$ & 2,361 & $-\quad 0,647$ & 3,008 & \\
\hline$-0,15$ & 1,610 & $-\quad 1,168$ & 2,778 & \\
\hline$-0,20$ & 1,259 & $-\quad 1,841$ & 3,100 & \\
\hline$-0,25$ & 1,047 & $-\quad 2,825$ & 3,873 & \\
\hline$-0,30$ & 0,899 & $-4,623$ & 5,522 & \\
\hline$-0,35$ & 0,783 & $-9,346$ & 10,129 & C \\
\hline 0,40 & 0,652 & $-72,033$ & 72,684 & I \\
\hline$-0,45$ & 0,635 & 14,850 & 14,215 & D \\
\hline$-0,50$ & 0,568 & 7,113 & 6,545 & כ \\
\hline$-0,55$ & 0,511 & 4,802 & 4,291 & כ \\
\hline$-0,60$ & 0,463 & 3,687 & 3,224 & \\
\hline$-0,65$ & 0,421 & 3,020 & 2,599 & $D$ \\
\hline$-0,70$ & 0,383 & 2,573 & 2,190 & \\
\hline$-0,75$ & 0,354 & 2,254 & 1,900 & \\
\hline$-0,80$ & 0,330 & 2,012 & 1,682 & \\
\hline$-0,85$ & 0,314 & 1,824 & 1,510 & \\
\hline$-0,90$ & 0,237 & 1,598 & 1,360 & \\
\hline$-1,00$ & 0,185 & 1,362 & 1,177 & \\
\hline
\end{tabular}

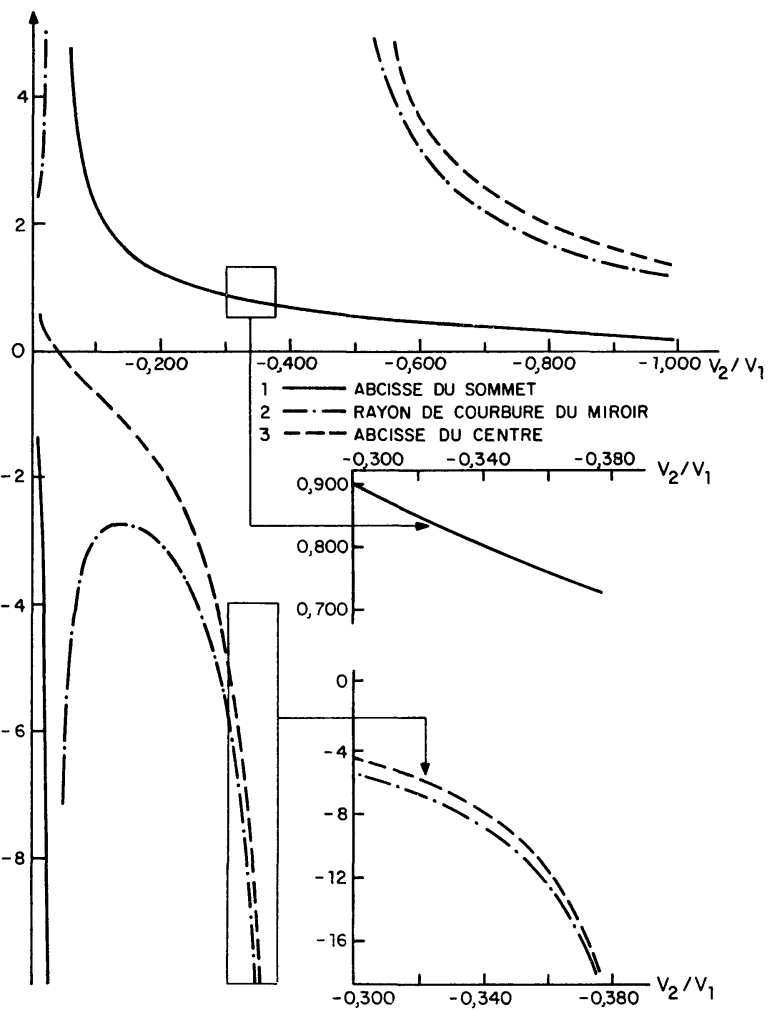

Fig. 3. - Propriétés optiques du miroir décrites à l'aide de l'abcisse du sommet, de l'abscisse du centre et du rayon de courbure du miroir. Les abscisses sont mesurées à partir de milieu de l'intervalle $e$ entre les deux cylindres.

[Optical properties of the mirror described using three parameters : abscissa of the summit, abscissa of the center, radius of curvature of the mirror. The abscissa are measured from the middle of the spacing between the cylinders.] l'abscisse $z=0$, véritable point de repère physique de cet élément. Le tableau II donne la valeur de ces coefficients. Le déterminant de la matrice formée par les coefficients $A_{0}, B_{0}, C_{0}, D_{0}$ de même que le déterminant de celle formée par $A_{\mathrm{m}}, B_{\mathrm{m}}, C_{\mathrm{m}}, D_{\mathrm{m}}$ vaut -1 .

\section{TABleau II}

Coefficients de la matrice du miroir bicylindre (ramenés au plan $z=0$ ) en fonction du rapport $V_{2} / V_{1}$. On rappelle : $D_{0}=-A_{0}$

\begin{tabular}{ccrr}
$V_{2} / V_{1}$ & \multicolumn{1}{c}{$A_{0}$} & \multicolumn{1}{c}{$B_{0}$} & \multicolumn{1}{c}{$C_{0}$} \\
$-\overline{0,01}$ & $-\overline{0,331}$ & $-\overline{1,069}$ & $-\overline{0,838}$ \\
$-0,05$ & $-0,971$ & 0,228 & 0,275 \\
$-0,10$ & $-0,570$ & 1,016 & 0,665 \\
$-0,15$ & $-0,159$ & 1,354 & 0,720 \\
$-0,20$ & 0,188 & 1,497 & 0,645 \\
$-0,25$ & 0,459 & 1,532 & 0,516 \\
$-0,30$ & 0,675 & 1,511 & 0,362 \\
$-0,35$ & 0,845 & 1,458 & 0,197 \\
$-0,40$ & 0,982 & 1,388 & 0,028 \\
$-0,45$ & 1,089 & 1,309 & $-0,141$ \\
$-0,50$ & 1,173 & 1,224 & $-0,305$ \\
$-0,55$ & 1,238 & 1,139 & $-0,466$ \\
$-0,60$ & 1,287 & 1,063 & $-0,620$ \\
$-0,65$ & 1,324 & 0,987 & $-0,770$ \\
$-0,70$ & 1,350 & 0,911 & $-0,913$ \\
$-0,75$ & 1,372 & 0,851 & $-1,052$ \\
$-0,80$ & 1,392 & 0,803 & $-1,189$
\end{tabular}

Ce test a permis de déceler d'éventuelles erreurs dans le calcul des trajectoires. Enfin notons que la cote du point de rebroussement pour les trajectoires paraxiales est très voisine de la cote $z_{\mathrm{n}}$ pour laquelle le potentiel est nul sur l'axe soit :

$$
z_{\mathrm{n}}=-\frac{1}{2 \omega} \ln \left(-\frac{V_{2}}{V_{1}}\right)
$$

Kel'man, Fedulina et Yadushev [5] ont étudié analytiquement les caractéristiques et les aberrations des miroirs cylindriques. Ils mentionnent bien en fonction du rapport des potentiels appliqués aux cylindres, les trois zones de fonctionnement. Mais les valeurs numériques des caractéristiques diffèrent assez sensiblement de celles que nous avons trouvées. Les auteurs font référence au travail de Recknagel et l'utilisation de l'approximation parabolique du potentiel sur de grands intervalles peut très bien expliquer l'ordre de grandeur des différences trouvées entre leurs résultats et les nôtres qui sont confirmés par l'expérience, comme on le verra en fin d'exposé.

2. Le spectromètre de masse magnétique à multiples passages $\left(\mathrm{SM}^{3} \mathbf{P}\right)$. - 2.1 GÉNÉRALITÉS. - Obtenir simultanément un bon pouvoir de résolution, une grande luminosité et un faible encombrement d'un spectromètre de masse magnétique semble, à première vue, irréalisable. L'utilisation de plusieurs appareils en 
succession ayant une bonne optique permet d'obtenir les deux premières qualités mais au détriment de la troisième. L'utilisation de miroirs et le fait que les trajectoires dans un champ magnétique sont distinctes lorsque l'on inverse le sens de parcours permet d'atteindre le triple objectif proposé plus haut. La figure 4 montre l'exemple d'un système à trois passages

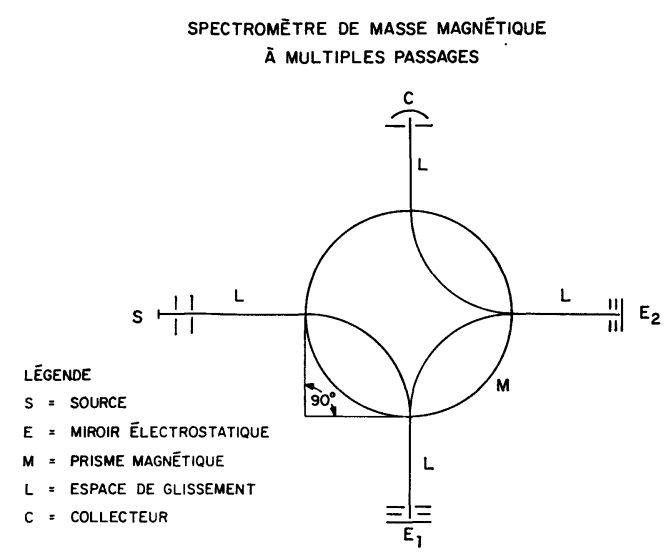

Fig. 4. - Exemple d'un spectromètre de masse magnétique à multiples passages. Dans ce cas particulier, la déflexion est de $90^{\circ}$ et 3 passages sont possibles.

[One example of the multipassage magnetic mass spectrometer. In this case the angle of deflexion is $90^{\circ}$ and there are three successive passages.]

où l'angle de déflexion est de $90^{\circ}$. Le choix du rayon de courbure des trajectoires égal au rayon de courbure des pièces polaires pour cet angle de déflexion, a pour effet d'éliminer l'aberration d'ouverture du prisme magnétique. Les principes de base utilisant la technique matricielle ont été exposés dans la publication de Fortin et Baril [1]. Nous allons les rappeler sommairement.

2.2 CARACTÉRISTIQUe OPTIQUE DU $\mathrm{SM}^{3} \mathrm{P}$ (imagerie dans le plan radial). - Quatre variables caractérisent une particule et la trajectoire qui lui est liée ; ce sont : $r$ l'écart spatial par rapport à la trajectoire principale, $\theta$ l'écart angulaire de la trajectoire particulière par rapport à la trajectoire principale, $\varepsilon$ l'écart relatif d'énergie par rapport à la particule parcourant la trajectoire principale, $\delta$ l'écart relatif de masse par rapport à la particule parcourant la trajectoire principale. La matrice de transfert d'un élément optique de plan d'entrée « 0 » et de plan sortie « $\mathrm{i}$ » a la forme suivante

$$
\left(\begin{array}{c}
r_{\mathrm{i}} \\
\alpha_{\mathrm{i}} \\
\varepsilon_{\mathrm{i}} \\
\delta_{\mathrm{i}}
\end{array}\right)=\left(\begin{array}{cccc}
A & B & E & F \\
C & D & G & H \\
0 & 0 & 1 & 0 \\
0 & 0 & 0 & 1
\end{array}\right)\left(\begin{array}{c}
r_{0} \\
\theta_{0} \\
\varepsilon_{0} \\
\delta_{0}
\end{array}\right)
$$

si les plans « 0 » et « $\mathrm{i}$ » sont au même potentiel. Choisissant des éléments d'optique qui forment ensemble un tout qui peut se répéter un certain nombre de fois, on peut atteindre le but visé. En particulier, on cher- chera à obtenir une matrice de transfert globale pour un ensemble répétitif de la forme

$$
\left(\begin{array}{cccc}
1 & 0 & E & F \\
0 & 1 & G & H \\
0 & 0 & 1 & 0 \\
0 & 0 & 0 & 1
\end{array}\right)
$$

La raison d'un tel choix est fort simple. La répétition $n$ fois d'un tel ensemble peut être représentée par la puissance $n$ de cette matrice qui vaut

$$
\left(\begin{array}{cccc}
1 & 0 & n E & n F \\
0 & 1 & n G & n H \\
0 & 0 & 1 & 0 \\
0 & 0 & 0 & 1
\end{array}\right)
$$

;. Dans un tel système afocal, les conditions de conjugaison sont conservées et la dispersion s'accroît avec le nombre de passages. Le grandissement étant unitaire, l'image de la source conserve alors la même dimension tout au cours des $n$ passages.

La matrice de transfert d'un passage peut être subdivisée en deux sous-ensembles, l'un relié au prisme l'autre au miroir. L'image formée par l'effet lentille du prisme magnétique sert d'objet pour le miroir qui produit une image jouant le rôle d'objet pour le prisme dans le passage suivant. On peut écrire les matrices des sous-ensembles sous la forme :

$$
\begin{aligned}
T_{\text {prisme }} & =\left(\begin{array}{cccc}
M_{\mathrm{p}} & 0 & E & F \\
-\frac{1}{F_{\mathrm{p}}} & \frac{1}{M_{\mathrm{p}}} & G & H \\
0 & 0 & 1 & 0 \\
0 & 0 & 0 & 1
\end{array}\right) \\
T_{\text {miroir }} & =\left(\begin{array}{cccc}
M_{\mathrm{m}} & 0 & 0 & 0 \\
\frac{1}{F_{\mathrm{m}}} & \frac{1}{M_{\mathrm{n}}} & 0 & 0 \\
0 & 0 & 1 & 0 \\
0 & 0 & 0 & 1
\end{array}\right)
\end{aligned}
$$

dont le produit est :

$$
\left(\begin{array}{cccc}
M_{\mathrm{p}} M_{\mathrm{m}} & 0 & E M_{\mathrm{m}} & F M_{\mathrm{m}} \\
\frac{M_{\mathrm{p}}}{F_{\mathrm{m}}}-\frac{F_{\mathrm{p}}}{M_{\mathrm{p}}} & \frac{1}{M_{\mathrm{m}}} M_{\mathrm{p}} & \frac{E}{F_{\mathrm{m}}}+\frac{F}{M_{\mathrm{m}}} & \frac{F}{F_{\mathrm{m}}}+\frac{G}{F_{\mathrm{p}}} \\
0 & 0 & 1 & 0 \\
0 & 0 & 0 & 1
\end{array}\right) .
$$

Si on impose les contraintes suivantes aux éléments du système

$$
\begin{aligned}
M_{\mathrm{m}} & =\frac{1}{M_{\mathrm{p}}} \\
F_{\mathrm{m}} & =F_{\mathrm{p}},
\end{aligned}
$$


on trouve alors la forme engendrée par un système afocal à grandissement unitaire.

De plus si l'on choisit $M_{\mathrm{m}}=1 / M_{\mathrm{p}}=-1$, l'image intermédiaire se trouve située au sommet du miroir. Le choix de $F_{\mathrm{m}}=F_{\mathrm{p}}$ n'est pas essentiel pour produire un système à grandissement unitaire et dont la dispersion spatiale s'accroît linéairement avec le nombre de passages, il entraîne seulement la propriété supplémentaire pour le système d'être afocal. La dispersion angulaire en fonction de l'écart d'énergie $\varepsilon$ croît non plus seulement linéairement le nombre de passages mais suivant l'expression

$$
\frac{n(n+1)}{2} \frac{E}{F_{\mathrm{m}}}+n G M_{\mathrm{m}}
$$

qui augmente très rapidement avec $n$. Un accroissement non linéaire de la dispersion angulaire en fonction de l'écart de masse existe de façon similaire et les formules s'appliquent mutatis mutandis.

Nous indiquons pour le bénéfice du lecteur la forme de la matrice de transfert $\left(T_{\text {esp }}\right)$ pour un espace de glissement de longueur $l$ ainsi que celle désignée par $\left(T_{\text {prisme }}\right)$ et correspondant à un prisme magnétique circulaire à entrée normale dont l'angle de déflexion et le rayon de courbure de la trajectoire principale sont respectivement $\Phi$ et $R_{\mathrm{p}}$.

$$
\begin{aligned}
& T_{\text {esp }}=\left(\begin{array}{cccc}
1 & l & 0 & 0 \\
0 & 1 & 0 & 0 \\
0 & 0 & 1 & 0 \\
0 & 0 & 0 & 1
\end{array}\right) \\
& T_{\mathrm{prisme}}^{\Phi}=\left(\begin{array}{cccc}
\cos \Phi & R_{\mathrm{p}} \sin \Phi & \frac{R_{\mathrm{p}}}{2}(1-\cos \Phi) & \frac{R_{\mathrm{p}}}{2}(1-\cos \Phi) \\
\frac{-\sin \Phi}{R_{\mathrm{p}}} & \cos \Phi & \frac{1}{2} \sin \Phi & \frac{1}{2} \sin \Phi \\
0 & 0 & 1 & 0 \\
0 & 0 & 0 & 1
\end{array}\right) .
\end{aligned}
$$

Dans le cas où $\Phi=90^{\circ}$ cette matrice se réduit à

$$
T_{\text {prisme }}^{90 \circ}=\left(\begin{array}{cccc}
0 & R_{\mathrm{p}} & \frac{R_{\mathrm{p}}}{2} & \frac{R_{\mathrm{p}}}{2} \\
-\frac{1}{R_{\mathrm{p}}} & 0 & \frac{1}{2} & \frac{1}{2} \\
0 & 0 & 1 & 0 \\
0 & 0 & 0 & 1
\end{array}\right) .
$$

On obtient un système conjugué de grandissement 1 si l'on fait précéder et suivre le prisme par des espaces de glissement de longueur égale à $R_{\mathrm{p}}$. Les informations concernant l'imagerie du miroir ont été données au paragraphe 1.4. Le lecteur dispose donc de tous les éléments nécessaires au calcul d'un système complet.

2.3 LE TRANSPORT DU FAISCEAU (étude de l'acceptance dans le plan transversal). - Contrairement au plan radial où luminosité et pouvoir de résolution sont intimement liés par l'intermédiaire de la largeur de la fente source, le plan transversal ne joue aucun rôle dans l'obtention d'un bon pouvoir de résolution. En l'absence de toute focalisation dans le plan transversal un $\mathrm{SM}^{3} \mathrm{P}$ à $n$ passages est équivalent à un spectromètre à un passage de rayon égal à $n$ fois celui d'un seul passage, la dimension de l'entrefer demeurant constante. L'acceptance, et en conséquence la transmission est réduite par un facteur $n$. La figure 5 montre l'allure de l'acceptance dans le plan transversal pour deux et trois passages. Dans cette figure, $h$ représente la dimension de l'entrefer, $R$ le rayon de courbure de la trajectoire principale, $\Phi$ l'angle de déflexion exprimé en radian et $k R$ la somme des espaces de glissement antérieur et postérieur au prisme. On voit que l'acceptance décroît au fur et à mesure que $n$ croît. Au contraire, s'il existe une certaine focalisation dans le plan transversal, il est possible de garantir qu'une certaine fraction de l'acceptance sera conservée quel que soit le nombre $n$ de passages. Cette fraction dépend de la matrice de transfert dans le plan transversal de l'unité répétitive du $\mathrm{SM}^{3} \mathrm{P}$ et de la forme du diagramme d'acceptance dans le prisme due à l'entrefer.

La technique des ellipses d'acceptance, utilisée pour le calcul du transport des faisceaux dans les grands accélérateurs $[6,7]$ nous sera d'un précieux support. Soient $l_{1}$ la distance parcourue par le faisceau entre l'entrée dans le prisme et l'entrée dans le miroir, $l_{2}$ la distance de la sortie du miroir jusqu'à l'entrée dans le 


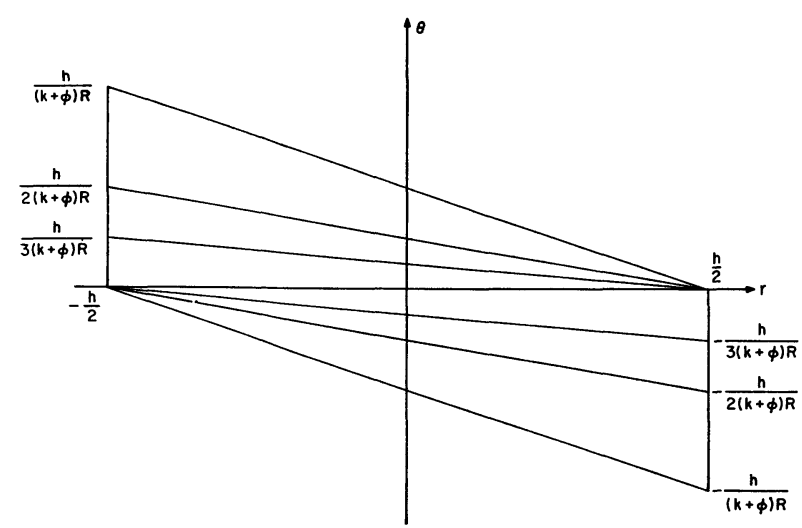

FIG. 5. - Schéma montrant l'acceptance d'un $\mathbf{S M}^{3} \mathbf{P}$ sans focalisation. La fente source et la fente détectrice sont de hauteur $h$. La longueur de parcours d'un passage est $(\Phi+k) R$ où $\Phi$ est l'angle de déflexion et $k R$ la longueur des espaces de glissement antérieur et postérieur au prisme.

[Schematic showing the acceptance of a $\mathrm{M}^{3} \mathrm{SP}$ without transverse focusing. Both source and detector slit have a height measured by $h$. The length of the main trajectory in one passage is $(\Phi+k) R$ where $\Phi$ is the deflexion angle and $k R$ is the length of the free space before and after the magnetic prism.]

prisme et $T_{\mathrm{m}}$ la matrice de transfert du miroir entre son entrée et sa sortie, mise sous la forme

$$
T_{\mathrm{m}}=\left(\begin{array}{ll}
A & B \\
C & D
\end{array}\right) \text {. }
$$

La matrice de transfert de l'unité de base de $\mathrm{SM}^{3} \mathrm{P}$ entre deux entrées successives dans le prisme est alors donnée par

$$
T_{\text {unité }}=\left(\begin{array}{cc}
1 & l_{2} \\
0 & 1
\end{array}\right)\left(\begin{array}{ll}
A & B \\
C & D
\end{array}\right)\left(\begin{array}{cc}
1 & l_{1} \\
0 & 1
\end{array}\right)=\left(\begin{array}{ll}
A^{\prime} & B^{\prime} \\
C^{\prime} & D^{\prime}
\end{array}\right) .
$$

Si $A^{\prime}+D^{\prime}$ est inférieur à deux, le faisceau pourra être conservé en partie quel que soit le nombre de passages. Au contraire si la trace est supérieure à deux le faisceau divergera de plus en plus. D'autre part il existe un type d'ellipse, définie par son orientation par rapport au diagramme d'acceptance et son rapport d'axe, qui jouit de la propriété de se répéter indéfiniment après chaque passage dans un système optique décrit par la matrice $A^{\prime}, B^{\prime}, C^{\prime}, D^{\prime}$. Il s'agit donc de trouver la surface de l'ellipse qui s'inscrit dans le parallépipède d'acceptance construit d'après les contraintes de l'entrefer du prisme.

L'étude de l'acceptance en d'autres points situés à l'intérieur du prisme ne semble pas nécessaire puisque la constante exprimée par rapport à l'entrée tient compte de l'ensemble du prisme.

3. Optimisation de la transmission. -3.1 . PosITION DU PROBLÈME. - Les deux données que nous nous fixons a priori sont :

- le rayon $R_{\mathrm{p}}$ du prisme magnétique,

- le rayon $\rho$ des cylindres.

ce dernier étant choisi, compte tenu de la géométrie du système à respecter, le plus grand possible afin de diminuer au maximum les aberrations.

Il reste à déterminer deux autres caractéristiques du miroir bicylindrique : sa position définie par celle du point $\mathrm{O}$ et le rapport $V_{2} / V_{1}$ des potentiels appliqués aux deux cylindres. Le choix de ces caractéristiques doit répondre aux deux impératifs qui ressortent de l'exposé fait au paragraphe précédent.

Le comportement de l'unité optique dans le plan radial est responsable du pouvoir de résolution. Nous venons de voir qu'il était souhaitable d'obtenir un grandissement unitaire. Pour qu'il en soit ainsi, la source ionique est placée à une distance égale au rayon $R_{\mathrm{p}}$ en avant du prisme magnétique. L'image $\mathrm{S}$, qu'en donne le prisme pour une certaine induction sera placée à une distance $R_{\mathrm{p}}$ après la sortie $\mathrm{B}$ du prisme et aura subi une rotation de $90^{\circ}$ telle que montrée sur la figure 6A. Le grandissement est unitaire. Il suffit alors

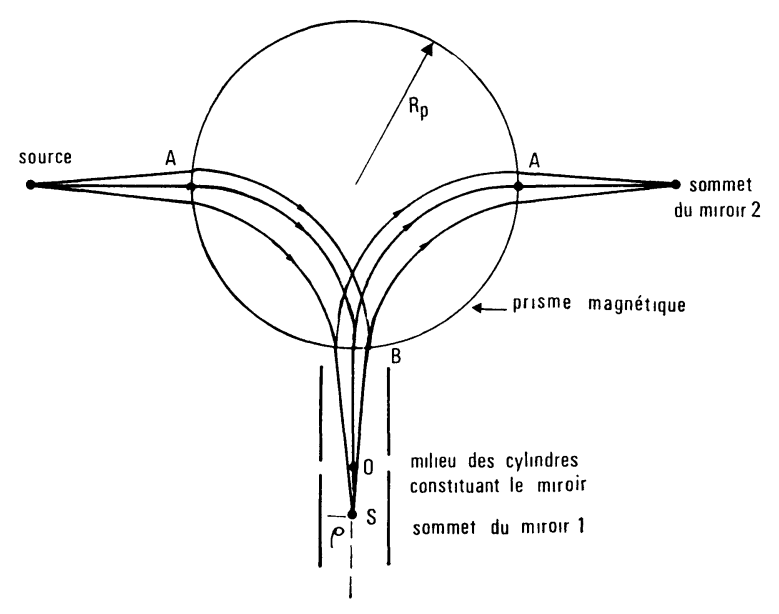

A. - PLAN RADial.

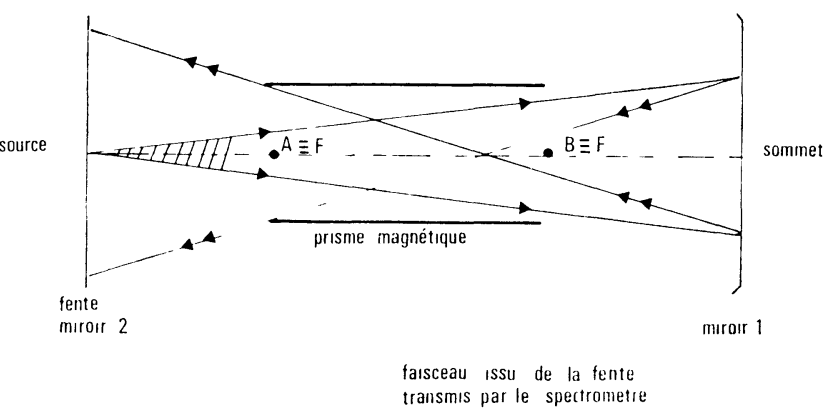

B. - Plan transVersal.

Fig. 6. - Description de l'unité optique et de ses éléments. Dans le plan radial la source s'image au sommet du miroir. Dans le plan transversal on a les espaces de glissement AO et OB et la zone miroir OSO.

[Schematic describing the optical unit and its component. In the radial plane, imaging of the source is made at the summit of the mirror. In the transversal plane there are free spaces AO and $\mathrm{OB}$ and the mirror region OSO.]

de faire coïncider cette image intermédiaire $S$ avec le sommet du miroir sphérique équivalent au miroir bicylindre. 
La luminosité du système est liée au comportement de l'unité optique dans le plan transversal. Dans ce plan le faisceau ionique issu de la source est divergent, l'unité doit donc lui donner une certaine convergence. L'idée première était de faire correspondre la source ionique située à $3,57 R_{\mathrm{p}}$ du sommet du miroir avec le centre du miroir et de créer ainsi un système répétitif non seulement dans le plan radial mais également dans le plan transversal. En fait, nous étudierons du point de vue de la luminosité toute la zone de convergence du miroir pour trouver les conditions optimales de luminosité et nous examinerons le cas particulier où le rayon du prisme $R_{\mathrm{p}}$ est le double du rayon du miroir $R_{\mathrm{m}}$ pour lequel l'unité optique devient dans le plan radial un système afocal.

3.2 Principe du Calcul de l'acceptance. - Pour l'étude de la luminosité du système nous choisissons comme unité optique l'ensemble

$$
(\mathrm{AO})+(\mathrm{OSO})+(\mathrm{OB}) \text {. }
$$

(AO) étant l'espace de glissement allant de l'entrée $\mathrm{du}$ prisme au repère géométrique $\mathrm{O}$ du miroir ;

(OSO) représentant la fonction miroir rapportée aux plans d'entrée et de sortie en $\mathrm{O}$;

(OB) étant l'espace de glissement, du point $O$ à l'entrée suivante dans le prisme magnétique (Fig. 6B).

Cette unité optique a comme matrice

$$
I_{\text {unité }}=\left(\begin{array}{ll}
A^{\prime} & B^{\prime} \\
C^{\prime} & D^{\prime}
\end{array}\right) \text {. }
$$

Pour que cette unité soit convergente, il faut que les valeurs des coefficients de la matrice soient telles que l'on puisse écrire $T_{\text {unité }}$ sous la forme

$$
\left(\begin{array}{ll}
\cos \mu+\mathrm{a} \sin \mu & \beta \sin \mu \\
-\gamma \sin \mu & \cos \mu-\alpha \sin \mu
\end{array}\right) .
$$

Dans ce cas, une particule émise dans les conditions $\left(\theta_{0}, r_{0}\right)$ sortira de la première unité optique avec les caractéristiques $\left(\theta_{\mathrm{i} 1}, r_{\mathrm{i} 1}\right)$ qui seront les conditions d'entrée de cette particule dans la deuxième unité optique. Ces conditions seront transformées en $\left(\theta_{\mathbf{i} 2}\right.$, $\left.r_{\mathrm{i} 2}\right)$. On montre que les diverses conditions $\left(\theta_{0}, r_{0}\right)$, $\left(\theta_{\mathrm{i} 1}, r_{\mathrm{i} 1}\right),\left(\theta_{\mathrm{i} 2}, r_{\mathrm{i} 2}\right) \ldots$ d'entrée d'une même particule dans les unités optiques successives satisfont à

$$
\gamma r^{2}+2 \alpha \theta r+\beta \theta^{2}=a b
$$

équation d'une ellipse de demi-axes $a$ et $b$.

A l'ensemble des conditions initiales $\left(\theta_{0}, r_{0}\right)$ des diverses particules émises par la source correspond une famille d'ellipses toutes semblables entre elles que l'on peut définir par

- le rapport de leurs axes

$$
\frac{a}{b}=H+\sqrt{H^{2}-1} \text { avec } H=\frac{1}{2}(\beta+\gamma) ;
$$

- l'inclinaison $\psi$ du grand axe sur l'axe des $r$ telle que

$$
\tan 2 \psi=-\frac{2 \alpha}{\beta-\gamma}
$$

En fait la présence du prisme magnétique impose une limitation aux conditions initiales des particules susceptibles de poursuivre leur course. Sur le graphe $(\theta, r)$, les conditions initiales permises par le prisme magnétique doivent être intérieures au parallélogramme dit d'acceptance représenté sur la figure 7 . Sur cette

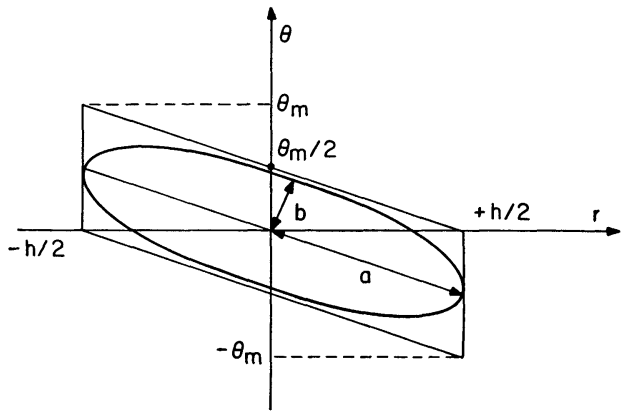

FIG. 7. - Ellipse et parallélogramme d'acceptance. Le parallélogramme est caractérisé par la demi-hauteur de l'entrefer $(h / 2)$ et l'angle maximal permis $\theta_{\mathrm{m}}$. L'ellipse d'acceptance est caractérisée par ses demi-axes $a$ et $b$; elle doit être inscrite à l'intérieur du parallélogramme.

[Acceptance ellipse and parallelogram. The parallelogram is defined by the half of the pole gap $(h / 2)$ and the maximal angle $\theta_{\mathrm{m}}$. The ellipse is caracterized by its half axes $a$ and $b$, and must fit inside the parallelogram.]

figure $h$ représente la hauteur de l'entrefer et $\theta_{\mathrm{m}}$ la valeur maximale de $\theta$, soit :

$$
\theta_{\mathrm{m}} \approx \tan \theta_{\mathrm{m}}=\frac{h}{1,57 R_{\mathrm{p}}},
$$

$1,57 R_{\mathrm{p}}$ étant le parcours des ions à l'intérieur du prisme. Pour qu'une particule de conditions initiales $\left(\theta_{0}, r_{0}\right)$ transmise par le prisme magnétique au premier passage, puisse l'être à tout passage, il faut que l'ellipse correspondante soit totalement interne au parallélogramme.

On est amené à déterminer l'ellipse de grandeur maximale inscrite dans le parallélogramme d'acceptance, cette ellipse étant appelée ellipse d'acceptance. En effet, la luminosité du système est proportionnelle à la surface de cette ellipse et elle est caractérisée par l'acceptance $\varepsilon$, telle que

$$
\varepsilon=a_{\max } \cdot b_{\max } .
$$

Pour déterminer $\varepsilon$, il suffit d'écrire que la valeur maximale que peut prendre $r$ est $r_{\mathrm{m}}=h / 2$. La géométrie des ellipses indique par ailleurs que $r_{\mathrm{m}}=\sqrt{\varepsilon \beta}$. On en déduit donc

$$
\varepsilon=\frac{1}{\beta} \cdot \frac{h^{2}}{4} .
$$

On vérifie ensuite que l'ellipse ainsi définie est bien contenue toute entière dans le parallélogramme et c est le cas dans ce qui suit.

Dans le cadre de ce travail, plutôt que de connaître la luminosité en valeur absolue, il nous semble plus intéressant d'évaluer la proportion de luminosité par rapport au maximum que l'on peut obtenir compte 
tenu de la présence du prisme magnétique. On a donc introduit l'acceptance relative

$$
\varepsilon_{\mathrm{r}}=\pi \frac{\varepsilon}{S}
$$

avec $S=$ surface du parallélogramme d'acceptance.

3.3 RÉSultats. - Le calcul des caractéristiques de l'ellipse d'acceptance, de l'acceptance $\varepsilon$ et de l'acceptance relative $\varepsilon_{\mathrm{r}}$ a été fait pour différents rapports $V_{2} / V_{1}$ et $R_{\mathrm{p}} / \rho$. Il n'est pas utile de faire varier la hauteur $h$ de l'entrefer car ce paramètre n'influe pas sur l'allure des courbes de $\varepsilon$ en fonction des deux rapports. Il peut tout au plus intervenir sur la valeur absolue.

La figure 8 représente les acceptances relatives en

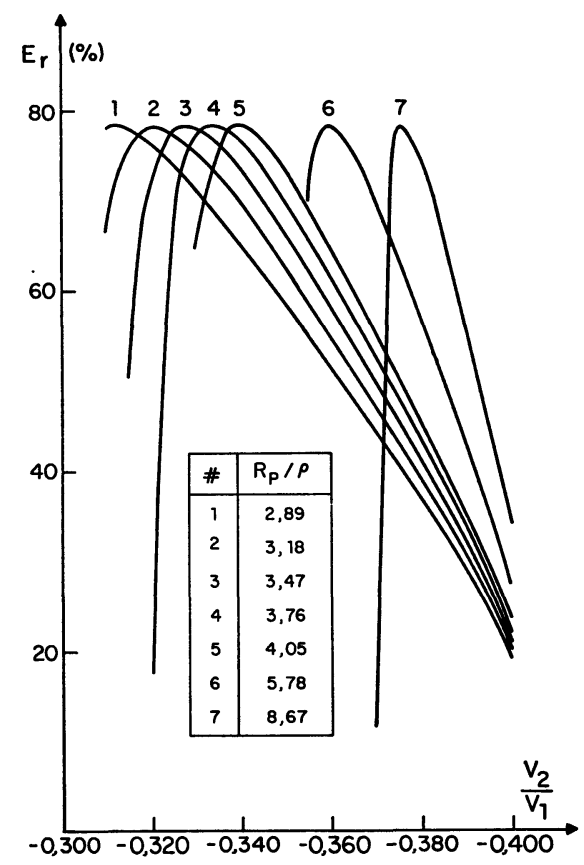

Fig. 8. - Acceptance relative en fonction de la tension appliquée sur le miroir et du rapport du rayon de courbure des trajectoires dans le prisme $R_{\mathrm{p}}$ sur le rayon des cylindres du miroir $\rho$.

[Relative acceptance as a function of the applied voltages on the mirror. The ratio of the radius of curvature $R_{\mathrm{p}}$ of the trajectories in the magnetic field to the radius $\rho$ of the mirror cylinders is the parameter.]

fonction du rapport $V_{2} / V_{1}$ des potentiels appliqués aux deux cylindres pour divers rapports $R_{\mathrm{p}} / \rho$ (rayon du prisme/rayon des cylindres). A partir des résultats, on peut en tirer les conclusions suivantes qui, pour la plupart, apparaissent immédiatement sur la figure 8 .

- La zone de convergence de l'ensemble correspond à une gamme de rapport $V_{2} / V_{1}$ de plus en plus réduite au fur et à mesure que $R_{\mathrm{p}} / \rho$ devient plus grand. La limite inférieure de la zone $V_{2} / V_{1}$ augmente en effet avec $R_{\mathrm{p}} / \rho$, alors que la limite supérieure est la même pour tous les $R_{\mathrm{p}} / \rho$ et correspond au fonctionnement en mode miroir plan de l'unité choisie.

- Pour un rapport $R_{\mathrm{p}} / \rho$ donné, l'acceptance et l'acceptance relative passent par un maximum.

Le maximum de l'acceptance relative est le même quelle que soit la valeur de $R_{\mathrm{p}} / \rho$; il atteint la valeur de $78,4 \%$. Par contre, le maximum de $\varepsilon$ décroît quand $R_{\mathrm{p}} / \rho$ croît, ce qui est normal.

Des deux observations précédentes il résulte que le maximum de $\varepsilon_{\mathrm{r}}$ est d'autant plus aigu que $R_{\mathrm{p}} / \rho$ est plus grand.

- La troisième considération se rapporte aux valeurs de $\left(V_{2} / V_{1}\right)_{\mathrm{m}}$ correspondant au maximum de $\varepsilon_{\mathrm{r}}$ pour les différents rapports $R_{\mathrm{p}} / \rho$ étudiés. Le tableau III met en évidence le fait suivant : l'acceptance maximale correspond toujours à un rapport $V_{2} / V_{1}$ pour lequel le rayon du miroir sphérique équivalent est le double du rayon du prisme. Sur la figure 6B, répondant à cette condition pour laquelle les foyers des miroirs se trouvent aux extrémités du prisme magnétique, le faisceau réfléchi (miroir $1 \rightarrow$ miroir 2 ) est entièrement conservé dans les réflexions ultérieures et se retrouve identique à lui-même après un cycle de quatre unités optiques. Par ailleurs, l'acceptance relative maximale du système correspond à un fonctionnement en système afocal dans le plan radial.

4. Résultats expérimentaux. - Le dispositif expérimental permet de recueillir les ions émis par la source ionique après une, deux ou trois traversées du prisme magnétique séparées, lorsqu'elles sont multiples, par des réflexions obtenues au moyen d'un miroir bicylindre.

Les cylindres sont positionnés vis-à-vis du prisme magnétique de façon que le sommet du miroir soit très proche du point de focalisation obtenu dans le plan radial par traversée du prisme. Ce réglage est valable dans la zone de convergence intéressante, car la variation de l'abscisse OS du sommet est de l'ordre de $\pm 5 \%$ du rayon du prisme dans la gamme des rapports $V_{2} / V_{1}$ allant de $-0,310$ à $-0,400$.

TABLEAU III

$$
\left(V_{2} / V_{1}\right)_{\mathrm{m}} \text { et }\left(V_{2} / V_{1}\right)_{\mathrm{d}} \text { en fonction de } R_{\mathrm{p}} / \rho
$$

$\begin{array}{lcrrrrrrrr}R_{\mathrm{p}} / \rho & 2,89 & 3,179 & 3,468 & 3,757 & 4,046 & 4,335 & 4,624 & 5,78 & 8,67 \\ \left(V_{2} / V_{1}\right)_{\mathrm{m}} & -0,312 & -0,321 & -0,328 & -0,335 & -0,340 & -0,345 & -0,349 & -0,360 & -0,376 \\ \left(V_{2} / V_{1}\right)_{\mathrm{d}} & -0,306 & -0,315 & -0,323 & -0,329 & -0,335 & -0,340 & -0,345 & -0,357 & -0,375\end{array}$

$\left(V_{2} / V_{1}\right)_{\mathrm{m}}$ représente le rapport de potentiel pour lequel il y a maximum d'acceptance.

$\left(V_{2} / V_{1}\right)_{\mathrm{d}}$ représente le rapport de potentiel pour lequel le rayon du miroir est double de celui du prisme.

Si l'on considère ces deux valeurs calculées à $1 \%$ près, on peut conclure à une coïncidence. 
4.1 EXPÉRIMENTATION DU MIROIR. - Dans une première expérience, l'induction est réglée de manière à recueillir les ions de masse 18 et l'on se propose d'étudier le courant collecté après deux traversées du prisme en fonction du rapport $V_{2} / V_{1}$ des potentiels appliqués aux cylindres du miroir.

La figure 9 représente la courbe ainsi obtenue. On

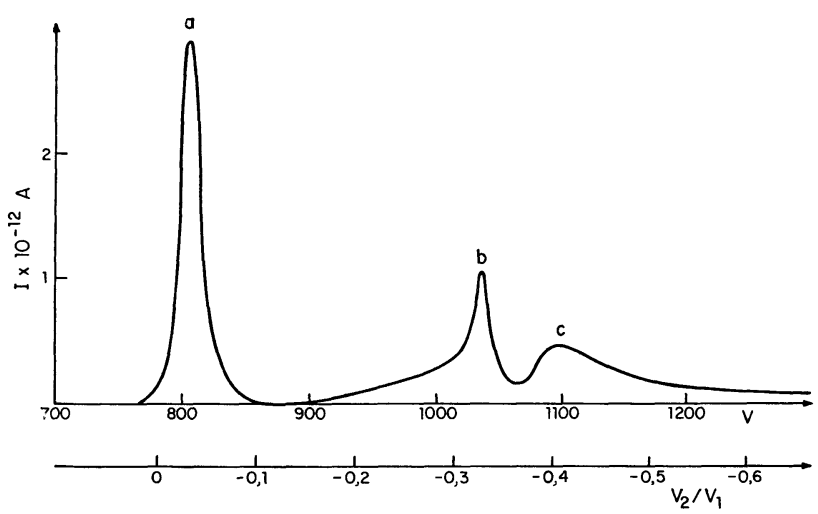

Fig. 9. - Intensité du pic 18 en fonction de la tension appliquée sur le miroir. Les trois pics correspondent respectivement aux situations suivantes : $a)\left(V_{2} / V_{1}=-0,037\right)$ le miroir et non le système est afocal. b) $\left(V_{2} / V_{1}=-0,328\right)$ l'unité optique est convergente dans le plan transversal et afocale dans le plan radial. c) $\left(V_{2} / V_{1}=-0,408\right)$, le miroir est alors équivalent à un miroir plan.

[Ion current of the 18 peak as a function of voltage applied to the mirror. The three maxima correspond respectively to the following operating conditions: $a)\left(V_{2} / V_{1}=-0.037\right)$ the mirror is afocal. $b)\left(V_{2} / V_{1}=-0.328\right)$ the optical unit is converging in the transversal plane and afocal in the radial plane. c) $\left(V_{2} / V_{1}=-0.408\right)$ the mirror become a plane mirror.]

peut y observer trois maxima correspondant à trois fonctionnements typiques particuliers du miroir.

Le premier maximum le plus intense a lieu pour $V_{2} / V_{1}=-0,037$. Pour ce rapport, le miroir est afocal ; son sommet est rejeté à l'infini tandis que son centre est à une distance fixe non confondue avec le point de focalisation produit par le prisme.

Le deuxième maximum a lieu pour $V_{2} / V_{1}=-0,328$, rapport pour lequel l'unité optique est convergente dans le plan transversal et afocale dans le plan radial, correspondant au maximum d'acceptance.

Le troisième maximum correspond à $V_{2} / V_{1}=-0,408$ Le miroir est alors équivalent à un miroir plan, le sommet du miroir se trouvant comme dans le cas précédent confondu avec l'image ionique donnée par la première traversée du prisme.

Les spectres obtenus par balayage en induction pour ces trois rapports et représentés figure 10 viennent confirmer ces résultats.

4.2 EFFETS SUR LES SPECTRES DE LA MULTIPLICATION DES PASSAGES IONIQUES A TRAVERS LE PRISME MAGNÉTIQUE. - La figure 11 représente trois spectres obtenus respectivement après un, deux et trois passages des ions à travers le prisme magnétique. La tension appliquée au miroir correspond à leur fonctionnement en
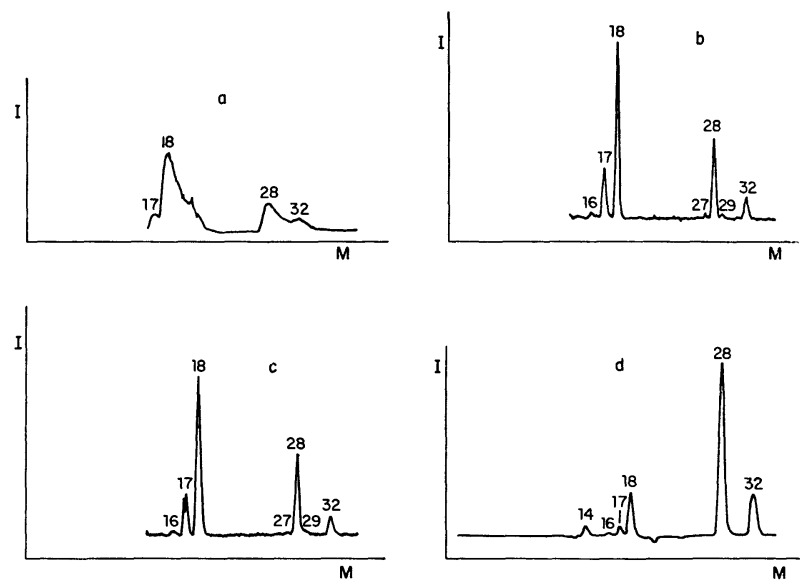

Fig. 10. - Allure des spectres de masse pour des tensions appliquées sur le miroir correspondant aux pics de la figure 9. a) Spectre correspondant au pic $a$ de la figure 9 (miroir afocal) ; $b$ ) Spectre correspondant au pic $b$ de la figure 9 (miroir convergent, système afocal dans le plan radial) :c) Spectre correspondant au pic $c$ dans la figure 9 (miroir plan); $d$ ) Spectre après un seul passage, alors que les précédents correspondent à deux passages.

[Various spectra corresponding to the maxima of the preceeding figure. $a$ ) Mass spectrum corresponding to maximum $a$ of figure 9. b) Mass spectrum corresponding to maximum $b$ of figure 9 ; c) Mass spectrum corresponding to maximum $c$ of figure 9 ; d) Mass spectrum after one passage (without mirror).]

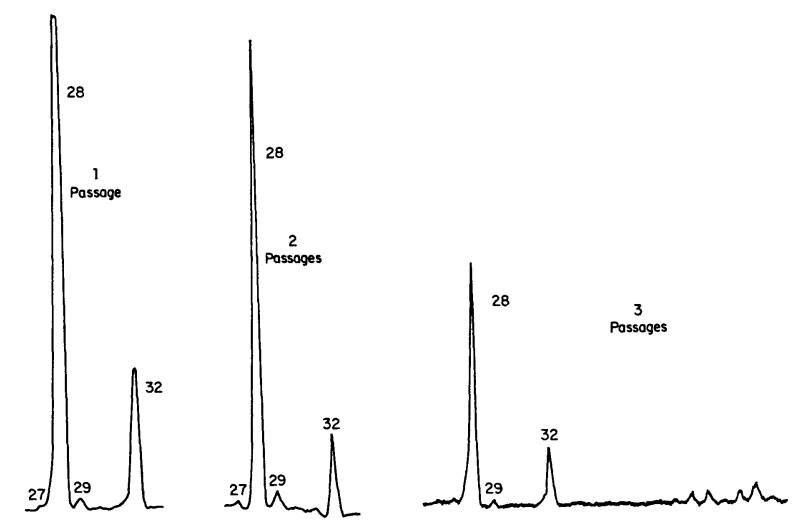

Fig. 11. - Spectres obtenus après un, deux, trois passages respectivement. Les spectres ayant été obtenus à des moments très différents, seul le pouvoir de résolution doit être comparé.

[Mass spectra obtained after one, two and three passages respectively. The spectra have been taken in very different conditions of gas composition and only the resolving power may be compared.]

miroir convergent réglé au maximum de transmission. Par ailleurs, il est à remarquer que les trois spectres n'ont pas été réalisés dans les mêmes conditions expérimentales, ce qui explique entre autres des proportions relatives des pics 18 et 28 fort diverses.

La considération de ces spectres conduit aux remarques suivantes. Tout d'abord le pouvoir de résolution est amélioré au fur et à mesure de l'augmentation du nombre de passages; ceci est particulièrement sensible au niveau de la séparation des pics 28 et 29 . Sur le dernier spectre, les spectres de masse voisins de 40 sont très nettement séparés les uns des autres, alors 
qu'un seul passage ne permet une séparation complète que jusqu'aux masses 20 . La seconde observation a trait à l'affaiblissement du faisceau. Bien que les conditions actuelles d'expérimentation ne nous permettent pas pour l'instant de faire les trois types de spectre dans des conditions expérimentales identiques, on peut en tirer cependant des renseignements qualitatifs sur l'intensité du faisceau. Un affaiblissement sensible de celui-ci a lieu lorsque l'on passe de un à deux passages. Par contre, il semblerait que le troisième passage n'atténue pas de façon appréciable l'intensité du faisceau. Ce dernier résultat est intéressant car il confirme que l'augmentation du pouvoir de résolution par multiplication des passages ne se fait pas au détriment de la luminosité.
Conclusion et remerciements. - Les travaux exposés dans cet article montrent que le miroir bicylindrique est un élément optique fort utile dans la mise au point d'un spectromètre de masse magnétique à multiples passages. En particulier, on doit noter que la luminosité demeure sensiblement constante après le deuxième passage. Des travaux devront être réalisés pour connaître l'existence de la source et la raison des pertes lors de la première réflexion. De même, des recherches sur la correction des aberrations devront être entreprises si on vise à multiplier le nombre de passages.

Nous tenons à souligner que ces travaux ont été réalisés au cours de séjours à l'université Laval de l'un des auteurs (C. Berger) dans le cadre des échanges Franco-Québécois.

\section{Bigliographie}

[1] Fortin, M., BARIL, M., Rev. Sci. Instrum. 43 (1972) 1140.

[2] Weber, C., Philips Tech. Rev. 24 (1963) 130.

[3] Recknagel, A., Z. Phys. 104 (1936) 381.

[4] El-Kareh, A. B., El-Kareh, J. C. J., Electron Beam, lenses and Optics, Vol. 1 (Academic Press, New York) 1970.

[5] Kel'man, V. M., Fedulina, L. V. et Yakushev, E. M., Zh. Tekh. Fiz. 41 (1971) 1489, Zh. Tekh. Fiz. 41 (1971) 1832.

[6] Fronteau, J., Chapitre 5.4, dans Focusing of Charged Particles (A. Septier, Academic Press New York) 1967.

[7] Bovet, C., Gourian, R., Gumowski, I., Reich, K. H., A. Selection of Formula and Data Useful for the Design of A. G. Synchrotrons. CERN/MPS-SI/Int. DL/70/4 (1970). 